\title{
Efficient Equalization of Time-Varying Channels in MIMO OFDM Systems
}

\author{
François Rottenberg, Member, IEEE, Xavier Mestre, Senior Member, IEEE, \\ François Horlin, Member, IEEE, and Jérôme Louveaux, Member, IEEE
}

\begin{abstract}
The orthogonality of the cyclic prefix orthogonal frequency division multiplexing (CP-OFDM) modulation is ensured as long as the channel can be assumed constant across the duration of one CP-OFDM symbol period. Unfortunately, this assumption may not hold anymore for a large variety of emerging scenarios with mobility, high carrier frequency and multiple carrier frequency offsets. To tackle this issue, we propose a novel equalization structure. In contrast to existing works in the literature, the equalizer is obtained by considering a Taylor approximation of the ideal time-varying channel equalizer function. This results in an extremely simple implementation only consisting of per-subcarrier multiplications and FFT/IFFT operations. The general form of the equalizer is particularized to two specific cases: zero forcing and linear minimum mean squared error. Furthermore, the implementation complexity of the equalizers is computed and an analytical formula is proposed to efficiently evaluate their performance. Finally, numerical results demonstrate the efficiency of the proposed receivers as compared to the ideal one and previous works.
\end{abstract}

Index Terms-OFDM, equalization, time-varying channels, multiple CFOs, massive MIMO.

\section{INTRODUCTION}

Wireless communication channels are inherently selective in both frequency and time domains, i.e., multipath propagation leads to frequency selectivity whereas mobility and carrier frequency offset (CFO) inherently results in time selectivity. Frequency selectivity is conventionally addressed by the use of multicarrier modulations, which typically accept simple per-subcarrier equalization. This is the case of cyclic prefix based orthogonal frequency division multiplexing (CPOFDM), which is the waveform universally employed in most recent wireless communications standards. The effect of time selectivity is usually neglected in this type of modulations, mainly because the channel can typically be assumed constant across the duration of a CP-OFDM symbol period.

These assumptions are no longer valid in recently considered high mobility scenarios, such as high speed trains or satel-

The research reported herein was partly funded by the Fonds pour la Formation à la Recherche dans l'Industrie et dans l'Agriculture, by the Belgian American Educational Foundation (B.A.E.F.) and by the Catalan and Spanish governments under grants 2017-SGR-01479 and RTI2018-099722-BI00 respectively.

François Rottenberg is with the University of Southern California, Los Angeles, U.S.A. (e-mail: frottenb@usc.edu).

François Rottenberg and Jérôme Louveaux are with the Universite catholique de Louvain, Louvain-la-Neuve, Belgium (e-mail: jerome.louveaux@uclouvain.be).

Xavier Mestre is with the Centre Tecnològic de Telecomunicacions de Catalunya, Barcelona, Spain (e-mail: xavier.mestre@cttc.cat).

François Horlin is with the Université libre de Bruxelles, Brussel, Belgium (e-mail: fhorlin@ulb.ac.be). lite extensions of the terrestrial components wireless systems. Additionally, the upcoming New Radio (NR) 5G standard introduces high frequency transmissions at millimeter waves (mmWave), for which the Doppler effects are much more significant than in conventional bands below $6 \mathrm{GHz}$. Furthermore, the recent development of massive MIMO architectures poses important challenges in the compensation of multiple CFOs. In the uplink, a large scale antenna array must demodulate the superposed transmission from multiple user equipments (UE), each one experimenting a different CFO. In the downlink, the use of cooperative communication architectures such as COoperative Multi-Point (COMP) require the demodulation of different symbol streams coming from multiple base stations (BS), which typically experiment different CFOs. Finally, the IEEE 802.11ax WiFi standard is expected to be released in 2019. While multi-user (MU) MIMO techniques were only available in downlink in previous IEEE 802.11ac standard, IEEE 802.11ax will allow to use MU MIMO in the uplink as well, which again leads to challenges in terms of CFO compensation. All these scenarios pose important challenges in terms of CP-OFDM signal demodulation under strong channel time selectivity.

There exist today a large body of literature that specifically targets the equalization of CP-OFDM signals in time selective channels. Most of the proposed solutions try to compensate the inter-carrier interference (ICI) that channel time variations originate in the frequency domain. In the presence of time selectivity, the equivalent channel matrix response after cyclic prefix removal and Fast Fourier Transform (FFT) is no longer diagonal, and this fact clearly leads to ICI. If the channel variations are mild enough, this matrix can be approximated as banded, and some specific methods that rely on this structure have been proposed in the literature. For example, [1] proposes to transform the approximate banded matrix into an extended block diagonal one, whereas [2] proposes an LDL factorization for band matrices in combination with a linear minimum mean squared error (LMMSE) equalizer. A variant of this method but implemented in the time domain is presented in [3] (see also [4] for a similar approach), whereas [5] proposes to use a time domain processor to enhance the band structure of the equivalent channel matrix after the receive FFT. This last approach is complemented in [6] with a low complexity block turbo-equalizer that exploits the banded nature of the equivalent channel matrix. An alternative approximation is given in [7], where a self-interference cancellation scheme is proposed to equalize the CP-OFDM signal for general doubly selective channels. 
There exists a number of approaches that model the time variations of the channel by truncating its representation in terms of a certain basis expansion. For example, [8] proposed the use of discrete prolate sequences in combination with an LMMSE equalizer, whereas [9] employed a complex exponential basis function in combination with the Viterbi or the BahlCocke-Jelinek-Raviv (BCJR) algorithms. On the other hand, [10] considered a general basis expansion and proposed an equalizer based on iterative methods that are commonly used to numerically solve systems of linear equations, whereas [11] and [12] respectively proposed a Cosine and Legendre basis expansion models for joint channel estimation and equalization using the Space Alternating Generalized Expectation Maximization (SAGE) algorithm. Other approaches try to approximate the channel variations using some parametric model. For example, the authors in [13] use a polynomial model to estimate the channel variations and then apply successive interference cancellation to remove the induced ICI.

A very interesting approach was proposed in [14] and later extended in [15], where a truncated Taylor series expansion is used for the time-varying channel frequency response. Using this truncated expansion in combination with a banded approximation of the channel matrix, [15] proposed a simplified LMMSE equalizer that could be complemented by a decisionfeedback receiver. Very similar approaches also exploiting the Taylor expansion of the channel frequency response were given in [16] and [17]. Recently, some equalizer structures for time varying channels have been proposed in [18], [19], which are based on the so-called partial FFT demodulation. The idea here consists in dividing the OFDM symbol time into shorter periods so that the channel can be approximated as constant on these shorter time spans. Then, a reduced size FFT is performed in these shorter time periods, and the result is properly combined by a linear processor, the coefficients of which are fixed to optimize the equalizer performance.

The above channel equalization algorithms have the problem that they are either based on ad-hoc approximations of the channel matrix (which is assumed to be essentially banded) or present a significant computational complexity (because they need matrix inversion, decoding, interference cancellation, etc.). In this paper, we propose an alternative approach that is somewhat similar to the Taylor series approximation in [15]. However, instead of considering the Taylor approximation of the channel frequency response, we propose to consider the Taylor approximation of the ideal time-varying channel equalizer. We will show that this will naturally lead to a family of CP-OFDM equalizers that are extremely simple to implement, mainly because they only require per-subcarrier processing and FFT/IFFT operations. Furthermore, our method offers a very simple way of controlling the quality of the equalizer output in terms of the number of terms of the Taylor approximation, so that one can a priori fix the number of stages so as to achieve a certain equalizer performance.

The rest of the paper is organized as follows. Section II presents the signal and channel model and introduces the Taylor approximation of the channel matrix. Section III presents the proposed channel equalizer in its general form, and particularizes its structure to some specific cases like ideal
LMMSE and zero forcing (ZF). This section also presents a complexity analysis of the presented algorithms, in terms of both computation of the equalizer coefficients and complexity of its implementation. Section IV then provides a theoretical analysis of the performance of the proposed algorithm that can be used to fix the number of equalizer stages in order to achieve a certain link quality. Finally, Section V presents a numerical validation of the proposed method and Section VI concludes the paper.

\section{A. Notations}

Vectors and matrices are denoted by bold lowercase and uppercase letters, respectively. Superscripts ${ }^{*},{ }^{T}$ and ${ }^{H}$ stand for conjugate, transpose and Hermitian transpose operators. The symbols tr, $\mathbb{E}, \Im$ and $\Re$ denote the trace, expectation, imaginary and real parts, respectively. $\jmath$ is the imaginary unit. The norm $\|\mathbf{A}\|$ is the Frobenius norm, i.e., $\|\mathbf{A}\|=$ $\sqrt{\operatorname{tr}\left(\mathbf{A}^{H} \mathbf{A}\right)} \cdot O(x)$ denotes a quantity that decays to zero at least as the same rate as $x$. $\mathbf{I}_{N}$ denotes the identity matrix of order $N . \otimes$ stands for the Kronecker product and $\delta_{n}$ is the Kronecker delta. The $\operatorname{diag}($.$) operator applied to a vector$ returns a diagonal matrix whose $k$-th diagonal entry is equal to the $k$-th entry of the argument vector.

\section{SySTEM MODEL}

\section{A. Transmission Model}

Let us denote by $M, N_{R}$ and $N_{T}$ the number of subcarriers, the number of transmit and receive antennas respectively. We assume pure spatial multiplexing so that the number of spatial streams is equal to $N_{T}$ with $N_{R} \geq N_{T}$. We assume that the cyclic prefix length is longer than the delay spread of the channel $L$, implying that successive OFDM multicarrier symbols are not interfering. For the sake of clarity and without loss of generality, we only consider the transmission of a single OFDM multicarrier symbol. In the same idea, we omit the effect of additive noise to focus on the interference induced by the time variations of the channel. Since the noise samples are not correlated with the data symbols, their effect can be studied independently, as will be shown in Section IV.

The OFDM symbol at antenna $j$, corresponding to one multicarrier symbol and before cyclic prefix insertion, is denoted by $\mathbf{s}_{j} \in \mathbb{C}^{M \times 1}$ and is equal to

$$
\mathbf{s}_{j}=\mathbf{F}^{H} \mathbf{d}_{j} \quad j=1, \ldots, N_{T},
$$

where $\mathbf{F} \in \mathbb{C}^{M \times M}$ is the unitary discrete Fourier transform matrix and $\mathbf{d}_{j} \in \mathbb{C}^{M \times 1}$ is the vector of transmitted symbols corresponding to spatial stream $j$. The transmit signal at each antenna is obtained after inserting the cyclic prefix.

We denote by $c_{i, j}[l, n]$ the time-varying channel impulse response between transmit antenna $j$, receive antenna $i$, corresponding to sampling time index $n$ and delay tap $l$ with $l=0, \ldots, L-1$. As a convention, we define the time samples $n=0$ and $n=M-1$ as the first and last samples of the multicarrier symbol before cyclic prefix insertion and after cyclic prefix removal. The received signal, after cyclic prefix removal, is defined as $r_{i}[n]$ for $n=0, \ldots, M-1$ and for 


$$
\begin{gathered}
\text { Structure of matrix } \mathbf{C}_{i, j} \in \mathbb{C}^{M \times M} \text { for the particular case } L=3 . \\
\mathbf{C}_{i, j}=\left(\begin{array}{cccccc}
c_{i, j}[0,0] & 0 & \ldots & 0 & c_{i, j}[2,0] & c_{i, j}[1,0] \\
c_{i, j}[1,1] & c_{i, j}[0,1] & 0 & \ldots & 0 & c_{i, j}[2,1] \\
c_{i, j}[2,2] & c_{i, j}[1,2] & c_{i, j}[0,2] & 0 & \ldots & 0 \\
0 & \ddots & \ddots & \ddots & & \vdots \\
\vdots & & c_{i, j}[2, n] & c_{i, j}[1, n] & c_{i, j}[0, n] & \vdots \\
\vdots & & \ddots & \ddots & \ddots & 0 \\
0 & \ldots & 0 & c_{i, j}[2, M-1] & c_{i, j}[1, M-1] & c_{i, j}[0, M-1]
\end{array}\right)
\end{gathered}
$$

receive antenna $i=1, \ldots, N_{R}$. Omitting the additive noise effect, we can write

$$
r_{i}[n]=\sum_{j=1}^{N_{T}} \sum_{l=0}^{L-1} c_{i, j}[l, n] s_{j}[(n-l) \bmod (M)],
$$

where $s_{j}[n]$ is the $n$-th entry of vector $\mathbf{s}_{j}$ and $\bmod (M)$ is the modulo operator. Defining $\mathbf{r}_{i}=\left(r_{i}[0], \ldots, r_{i}[M-1]\right)^{T} \in$ $\mathbb{C}^{M \times 1}$, we can write

$$
\mathbf{r}_{i}=\sum_{j=1}^{N_{T}} \mathbf{C}_{i, j} \mathbf{s}_{j}=\sum_{j=1}^{N_{T}} \mathbf{C}_{i, j} \mathbf{F}^{H} \mathbf{d}_{j} \quad i=1, \ldots, N_{R} .
$$

The structure of the matrix $\mathbf{C}_{i, j} \in \mathbb{C}^{M \times M}$ is described in (1) for the particular case $L=3$ and for arbitrary transmit and receive antennas $j$ and $i$. One should note that, unlike conventional OFDM under time-invariant channel, the matrix $\mathbf{C}_{i, j}$ is not circulant. Hence, taking the FFT at the receiver is not sufficient to convert the effect of the channel into a set of $M$ parallel frequency flat MIMO channels, free from intercarrier interference. This is critical as conventional receiver operations need to be revisited.

The demodulated symbols, after the FFT, at receive antenna $i$ are given by $\mathbf{z}_{i}=\mathbf{F} \mathbf{r}_{i} \in \mathbb{C}^{M \times 1}$. Stacking the vectors $\mathbf{r}_{i}$ and $\mathbf{z}_{i}$ for $i=1, \ldots, N_{R}$ gives

$$
\begin{aligned}
& \mathbf{r}=\mathbf{C}\left(\mathbf{I}_{N_{T}} \otimes \mathbf{F}^{H}\right) \mathbf{d}, \\
& \mathbf{z}=\left(\mathbf{I}_{N_{R}} \otimes \mathbf{F}\right) \mathbf{C}\left(\mathbf{I}_{N_{T}} \otimes \mathbf{F}^{H}\right) \mathbf{d},
\end{aligned}
$$

where we defined the notations $\mathbf{r} \in \mathbb{C}^{N_{R} M \times 1}, \mathbf{z} \in \mathbb{C}^{N_{R} M \times 1}$, $\mathbf{d} \in \mathbb{C}^{N_{T} M \times 1}$ and $\mathbf{C} \in \mathbb{C}^{N_{R} M \times N_{T} M}$ as

$$
\begin{aligned}
\mathbf{r} & =\left(\begin{array}{c}
\mathbf{r}_{1} \\
\vdots \\
\mathbf{r}_{N_{R}}
\end{array}\right), \mathbf{z}=\left(\begin{array}{c}
\mathbf{z}_{1} \\
\vdots \\
\mathbf{z}_{N_{R}}
\end{array}\right), \mathbf{d}=\left(\begin{array}{c}
\mathbf{d}_{1} \\
\vdots \\
\mathbf{d}_{N_{T}}
\end{array}\right), \\
\mathbf{C} & =\left(\begin{array}{ccc}
\mathbf{C}_{1,1} & \ldots & \mathbf{C}_{1, N_{T}} \\
\vdots & \ddots & \vdots \\
\mathbf{C}_{N_{R}, 1} & \ldots & \mathbf{C}_{N_{R}, N_{T}}
\end{array}\right) .
\end{aligned}
$$

\section{B. Channel Model}

A conventional OFDM processing, taking an FFT of the received signals at each antenna and performing per-subcarrier equalization, is not sufficient to compensate for the doubly selective nature of the channel. Instead, one could directly try to equalize the received signal $\mathbf{r}$ by equalizing the channel matrix C. However, this would have a prohibitive complexity burden as matrix $\mathbf{C}$ is of dimension $N_{R} M \times N_{T} M$. Here, we propose an alternative representation of matrix $\mathbf{C}$ that will prove useful in Section III to derive the structure of the proposed equalizer, leveraging the computational efficiency of FFT's and IFFT's and resulting in a much lower complexity of implementation. To do this, we first need to make assumptions on the channel variations in time, which will allow us to derive rigorous bounds on the residual error of the proposed approximations in Section III and IV.

(As1): The time-varying channel $c_{i, j}[l, n]$ comes from the evaluation of the analog channel $\tilde{c}_{i, j, l}(t)$ at sampling time $t=$ $\left(n-\frac{M}{2}\right) \frac{T}{M}$, where $T$ is the OFDM symbol period and the time $t=0$ is chosen as the middle of the OFDM symbol. The Doppler spectrum of each channel tap, defined as $\phi_{i, j, l}(\nu)$, is assumed to be compactly supported in $\left[-f_{d}, f_{d}\right]$ so that

$$
\tilde{c}_{i, j, l}(t)=\int_{-f_{d}}^{f_{d}} \phi_{i, j, l}(\nu) e^{\jmath 2 \pi \nu t} d \nu .
$$

One can note that the assumption of a compactly supported Doppler spectrum makes sense in practice. Indeed, channel variations are in practice bounded by physical constraints. When caused by CFOs, the channel Doppler spectrum is an atom at the corresponding frequency $f_{d}$, which is compactly supported by definition. The same can be said if the channel variations are caused by scatterer, transmitter or receiver mobility. As an example, denoting by $V$ the difference of speed between transmitter and receiver for static scatterers, the maximal Doppler shift is given by $f_{d}=V f_{c} / c[\mathrm{~Hz}]$ where $f_{c}$ is the carrier frequency and $c$ is the speed of light [20]. Since $V, c$ and $f_{c}$ are finite, it makes sense to assume that the Doppler spectrum has a limited support. As an example, the classical Jakes spectrum, defined as

$$
S(\nu)= \begin{cases}\frac{1}{\pi \sqrt{f_{d}^{2}-\nu^{2}}} & \text { if } \nu \in\left[-f_{d}, f_{d}\right] \\ 0 & \text { otherwise }\end{cases}
$$

satisfies (As1). Let us further define the vector $\mathbf{c}_{i, j}^{(r)} \in \mathbb{C}^{L \times 1}$ as

$$
\mathbf{c}_{i, j}^{(r)}=\left(\begin{array}{c}
c_{i, j}^{(r)}[0] \\
\vdots \\
c_{i, j}^{(r)}[L-1]
\end{array}\right), \quad c_{i, j}^{(r)}[l]=\left.\frac{d^{(r)}}{d t^{r}} \tilde{c}_{i, j, l}(t)\right|_{t=0},
$$


where the coefficient $c_{i, j}^{(r)}[l]$ is the $r$-th time derivative of the analog channel $\tilde{c}_{i, j, l}(t)$ evaluated at time $t=0$. We are now in the position to introduce the main result of this section.

Proposition 1. Under (As1) and for a certain OFDM symbol period $T$, matrix $\mathbf{C}$ can be written as $\mathbf{C}=\tilde{\mathbf{C}}(T)$, with function $\tilde{\mathbf{C}}(\tilde{T})$ defined as

$$
\tilde{\mathbf{C}}(\tilde{T})=\sum_{r=0}^{+\infty} \frac{\tilde{T}^{r}}{r !}\left(\mathbf{I}_{N_{R}} \otimes \mathbf{D}^{r}\right) \mathbf{C}^{(r)},
$$

where matrix $\mathbf{D} \in \mathbb{R}^{M \times M}$ is a real diagonal matrix whose $n$-th diagonal entry is given by $\left(\frac{n-\frac{M}{2}}{M}\right)$ and $\mathbf{C}^{(r)} \in$ $\mathbb{C}^{N_{R} M \times N_{T} M}$ has the following structure

$$
\mathbf{C}^{(r)}=\left(\begin{array}{ccc}
\mathbf{C}_{1,1}^{(r)} & \ldots & \mathbf{C}_{1, N_{T}}^{(r)} \\
\vdots & \ddots & \vdots \\
\mathbf{C}_{N_{R}, 1}^{(r)} & \cdots & \mathbf{C}_{N_{R}, N_{T}}^{(r)}
\end{array}\right)
$$

Each block matrix $\mathbf{C}_{i, j}^{(r)} \in \mathbb{C}^{M \times M}$ is circulant and has vector $\mathbf{\Sigma} \mathbf{c}_{i, j}^{(r)}$ as its first column, where $\boldsymbol{\Sigma} \in \mathbb{R}^{M \times L}$ is a zero padding matrix that appends $M-L$ zeros to $\mathbf{c}_{i, j}^{(r)}$, i.e., $\boldsymbol{\Sigma}=\left(\mathbf{I}_{L}, \mathbf{0}_{L \times M-L}\right)^{T}$.

Proof. The proof relies on a Taylor expansion of the channel with respect to its variations in time. See Appendix VII-A.

Proposition 1 introduces a dependence of $\tilde{\mathbf{C}}(\tilde{T})$ in an auxiliary variable $\tilde{T}$, even though $\mathbf{C}$ was obtained for a specific OFDM period $T$, i.e., $\mathbf{C}=\tilde{\mathbf{C}}(T)$. The reason for introducing the dependence in $\tilde{T}$ will become clearer in Section III. Note that, for a fixed number of subcarriers $M$, a variation of the OFDM period $\tilde{T}$ influences how the channel will have varied from the beginning to the end of the OFDM block. In other words, it quantifies how much the entries of each diagonal of $\mathbf{C}_{i, j}$ differ from one another. In the extreme theoretic case $\tilde{T}=0$, the OFDM symbol is concentrated in time, it does not suffer from the time variations of the channel and matrix $\mathbf{C}_{i, j}$ becomes circulant again, as in the time-invariant OFDM case. Another motivation for introducing the notation $\tilde{\mathbf{C}}(\tilde{T})$ is that it will allow us to define the derivatives of the channel with respect to the variable $\tilde{T}$ in Section III.

Moreover, one of the big advantages of using the Taylor expansion in Proposition 1 is that, as opposed to $\mathbf{C}_{i, j}$ described in (1), matrices $\mathbf{C}_{i, j}^{(r)}$ have a circulant structure, which can be exploited for efficient processing using combination of FFT's and IFFT's. As a consequence, we can write the eigenvalue decomposition of matrix $\mathbf{C}_{i, j}^{(r)}$ as $\mathbf{C}_{i, j}^{(r)}=\mathbf{F}^{H} \boldsymbol{\Lambda}_{i, j}^{(r)} \mathbf{F}$ where $\boldsymbol{\Lambda}_{i, j}^{(r)}=\operatorname{diag}\left(\sqrt{M} \mathbf{F} \boldsymbol{\Sigma} \mathbf{c}_{i, j}^{(r)}\right)$. Using this and the result of Proposition 1, we can rewrite $\mathbf{C}$ as

$$
\mathbf{C}=\tilde{\mathbf{C}}(T)=\sum_{r=0}^{+\infty} \frac{T^{r}}{r !}\left(\mathbf{I}_{N_{R}} \otimes \mathbf{D}^{r} \mathbf{F}^{H}\right) \boldsymbol{\Lambda}^{(r)}\left(\mathbf{I}_{N_{T}} \otimes \mathbf{F}\right)
$$

where $\Lambda^{(r)} \in \mathbb{C}^{N_{R} M \times N_{T} M}$ has the following structure made of diagonal matrices

$$
\boldsymbol{\Lambda}^{(r)}=\left(\begin{array}{ccc}
\boldsymbol{\Lambda}_{1,1}^{(r)} & \ldots & \boldsymbol{\Lambda}_{1, N_{T}}^{(r)} \\
\vdots & \ddots & \vdots \\
\boldsymbol{\Lambda}_{N_{R}, 1}^{(r)} & \ldots & \boldsymbol{\Lambda}_{N_{R}, N_{T}}^{(r)}
\end{array}\right) .
$$

We can also rewrite the demodulated symbols in (2) as

$$
\mathbf{z}=\sum_{r=0}^{+\infty} \frac{T^{r}}{r !}\left(\mathbf{I}_{N_{R}} \otimes \mathbf{F D}^{r} \mathbf{F}^{H}\right) \mathbf{\Lambda}^{(r)} \mathbf{d} .
$$

The expression in (7) gives an intuition of the impact of time-varying channels on the demodulated symbols $\mathbf{z}$. If the channel was time-invariant, we would have $\boldsymbol{\Lambda}^{(r)}=\delta_{r} \boldsymbol{\Lambda}^{(0)}$ and only the first term of the sum would be present, i.e., $\mathbf{z}=\boldsymbol{\Lambda}^{(0)} \mathbf{d}$. This is the conventional result of OFDM systems under static channel conditions. The demodulated symbols at each subcarrier propagate through a frequency flat MIMO channel. Note that the same result would be obtained as $T \rightarrow 0$. On the other hand, as the OFDM symbol duration $T$ increases or the channel varies faster in time (meaning that the amplitude of the entries of $\boldsymbol{\Lambda}^{(r)}$ are amplified for $r>0$ ), the demodulated symbols suffer from inter-carrier interference through the circulant mixing matrix $\mathbf{F D}^{r} \mathbf{F}^{H}$ and the channel derivatives in time $\boldsymbol{\Lambda}^{(r)}, r>0$.

Note that we here chose $T$ as the fundamental varying parameter. The impact of varying other system parameters can be inferred through the corresponding change of $T$. As an example, if, for a fixed bandwidth, $M$ increases, it will induce a smaller subcarrier spacing $1 / T$ and hence a larger $T$. As a result, the system would be more sensitive to time variations of the channel.

\section{Proposed Equalization Structure}

Examining (2) and (7), one can define the end-to-end transfer function $\mathbf{H} \in \mathbb{C}^{N_{R} M \times N_{T} M}$ as

$$
\begin{aligned}
\mathbf{H} & =\left(\mathbf{I}_{N_{R}} \otimes \mathbf{F}\right) \mathbf{C}\left(\mathbf{I}_{N_{T}} \otimes \mathbf{F}^{H}\right) \\
& =\sum_{r=0}^{+\infty} \frac{T^{r}}{r !}\left(\mathbf{I}_{N_{R}} \otimes \mathbf{F D}^{r} \mathbf{F}^{H}\right) \boldsymbol{\Lambda}^{(r)},
\end{aligned}
$$

so that $\mathbf{z}=\mathbf{H d}$. In order to recover the information symbol vector $\mathbf{d}$, one can use a linear equalizer $\mathbf{B} \in \mathbb{C}^{N_{T} M \times N_{R} M}$ and obtain a symbol estimate as $\hat{\mathbf{d}}=\mathbf{B z}$. Different criteria may be used to design the equalizer B. For instance, the choice

$$
\mathbf{B}=\left(\mathbf{H}^{H} \mathbf{H}+\lambda \mathbf{I}_{N_{T} M}\right)^{-1} \mathbf{H}^{H}
$$

gives the ZF equalizer if $\lambda=0$ and the MMSE equalizer if $\lambda$ is chosen as the inverse of the signal-to-noise ratio. It is clear that using this type of equalizer would be prohibitive in terms of complexity as it scales as $O\left(N_{T}^{3} M^{3}\right)$ due to the matrix inversion. Furthermore, one would have to implement a large matrix multiplication to equalize the received samples. Therefore, we propose an approximation of the ideal equalizer B, leveraging the efficiency of FFT's and IFFT's and allowing for MIMO processing at the subcarrier level. 


\section{A. Equalizer Structure}

Following the same formalism that we used to define the channel function $\tilde{\mathbf{C}}(\tilde{T})$, we first define the function $\tilde{\mathbf{H}}(\tilde{T})$ as the end-to-end transfer function from the transmitted symbols $\mathbf{d}$ to the demodulated symbols $\mathbf{z}$ for the auxiliary symbol period $\tilde{T}$ and so that $\mathbf{H}=\tilde{\mathbf{H}}(T)$, i.e.,

$$
\tilde{\mathbf{H}}(\tilde{T})=\sum_{r=0}^{+\infty} \frac{\tilde{T}^{r}}{r !}\left(\mathbf{I}_{N_{R}} \otimes \mathbf{F} \mathbf{D}^{r} \mathbf{F}^{H}\right) \mathbf{\Lambda}^{(r)} .
$$

Secondly, we can define the equalizer function $\tilde{\mathbf{B}}(\tilde{T})$ which depends on $\tilde{\mathbf{H}}(\tilde{T})$ and hence, on the auxiliary variable $\tilde{T}$. The ideal equalizer $\mathbf{B}$ can be viewed as the evaluation of $\tilde{\mathbf{B}}(\tilde{T})$ at $\tilde{T}=T$, i.e., $\mathbf{B}=\tilde{\mathbf{B}}(T)$. Furthermore, we introduce the following assumption on the smoothness of the equalizer function $\tilde{\mathbf{B}}(\tilde{T})$.

(As2): The equalizer function $\tilde{\mathbf{B}}(\tilde{T})$ is $C^{R+1}$ smooth for $\tilde{T} \in[0, T]$.

We denote by $\mathbf{B}^{(r)}$ the $r$-th order derivative of $\tilde{\mathbf{B}}(\tilde{T})$ evaluated at $\tilde{T}=0$, i.e.,

$$
\mathbf{B}^{(r)}=\left.\frac{d^{(r)}}{d \tilde{T}^{r}} \tilde{\mathbf{B}}(\tilde{T})\right|_{\tilde{T}=0} .
$$

Note that (As2) implies that the $R+1$ first derivatives are bounded. We can now write the expression of the proposed equalizer.

Theorem 1. Under (As2), an approximation of the ideal equalizer $\mathbf{B}=\tilde{\mathbf{B}}(T)$ of order $R$ with respect to the channel variations in time is given by

$$
\hat{\mathbf{B}}_{R}=\sum_{r=0}^{R} \frac{T^{r}}{r !} \mathbf{B}^{(r)}
$$

and, as $T \rightarrow 0$, the approximation error can be bounded as

$$
\left\|\mathbf{B}-\hat{\mathbf{B}}_{R}\right\|=O\left(T^{R}\right) .
$$

Proof. The proof is given in Appendix VII-B.

Theorem 1 provides an approximation of the transmitted symbols of order $R$ with respect to the channel variations in time. Let us denote these estimated symbols as $\hat{\mathbf{d}}_{R}$, defined as $\hat{\mathbf{d}}_{R}=\hat{\mathbf{B}}_{R} \mathbf{z}$.

\section{B. Practical Implementation}

This section aims at showing how to efficiently implement the proposed equalizers. To do this, we need to make a choice for the structure of the ideal equalizer $\tilde{\mathbf{B}}(\tilde{T})$. However, one should note that other general equalizer forms are also possible. Furthermore, we introduce the following notation that will prove useful to derive the expression of the equalizers

$$
\mathbf{H}^{(r)}=\left.\frac{d^{(r)}}{d \tilde{T}^{r}} \tilde{\mathbf{H}}(\tilde{T})\right|_{\tilde{T}=0}=\left(\mathbf{I}_{N_{R}} \otimes \mathbf{F} \mathbf{D}^{r} \mathbf{F}^{H}\right) \mathbf{\Lambda}^{(r)} .
$$

1) SISO, zero forcing: Let us first focus on the SISO case, $N_{R}=N_{T}=1$ and the zero forcing case, $\lambda=0$ so that the ideal equalizer function is set to $\tilde{\mathbf{B}}(\tilde{T})=(\tilde{\mathbf{H}}(\tilde{T}))^{-1} \in$ $\mathbb{C}^{M \times M}$ and matrix $\boldsymbol{\Lambda}^{(r)}$ is a $M \times M$ diagonal matrix. Using Theorem 1, the definition in (11) and the derivative rule $\frac{d \mathbf{Y}^{-1}}{d t}=-\mathbf{Y}^{-1} \frac{d \mathbf{Y}}{d t} \mathbf{Y}^{-1}$, we can find the different orders of approximation of the ideal equalizer $\mathbf{B}=\mathbf{H}^{-1}$.

The zero order approximation is simply given by

$$
\hat{\mathbf{B}}_{0}=\left(\mathbf{H}^{(0)}\right)^{-1}=\left(\boldsymbol{\Lambda}^{(0)}\right)^{-1},
$$

which corresponds to the conventional single-tap persubcarrier OFDM equalization. The first order approximation is given by

$$
\begin{aligned}
\hat{\mathbf{B}}_{1} & =\hat{\mathbf{B}}_{0}-\left(\mathbf{H}^{(0)}\right)^{-1} \mathbf{H}^{(1)}\left(\mathbf{H}^{(0)}\right)^{-1} \\
& =\hat{\mathbf{B}}_{0}-T\left(\boldsymbol{\Lambda}^{(0)}\right)^{-1} \mathbf{F} \mathbf{D} \mathbf{F}^{H} \boldsymbol{\Lambda}^{(1)}\left(\boldsymbol{\Lambda}^{(0)}\right)^{-1},
\end{aligned}
$$

and the second order approximation can be similarly obtained as

$$
\begin{aligned}
\hat{\mathbf{B}}_{2} & =\hat{\mathbf{B}}_{1}+T^{2}\left(\left(\boldsymbol{\Lambda}^{(0)}\right)^{-1} \mathbf{F} \mathbf{D} \mathbf{F}^{H} \boldsymbol{\Lambda}^{(1)}\right)^{2}\left(\boldsymbol{\Lambda}^{(0)}\right)^{-1} \\
& -\frac{T^{2}}{2}\left(\boldsymbol{\Lambda}^{(0)}\right)^{-1} \mathbf{F D}^{2} \mathbf{F}^{H} \boldsymbol{\Lambda}^{(2)}\left(\boldsymbol{\Lambda}^{(0)}\right)^{-1}
\end{aligned}
$$

The great advantage of the proposed equalizer $\hat{\mathbf{B}}_{R}$ is that it is extremely simple to implement. Indeed, the expression of the equalizers is only composed of diagonal matrices, which can be applied as scalar multiplications at the subcarrier level and FFT-IFFT pairs which only require $O(M \log (M))$ operations. Furthermore, no matrix inversions are necessary even though the proposed equalizer approximates the ideal channel inverting equalizer $\mathbf{B}=\mathbf{H}^{-1}$. Fig. 1 shows the block diagram of the proposed equalizer for $R=0$ (conventional OFDM receiver), $R=1$ and $R=2$. Note that the proposed equalizer was efficiently implemented combining the intermediate outputs of previous stages.

2) MIMO, MMSE: Let us now consider the ideal MIMO equalizer given in (9) so that the ideal equalizer function $\tilde{\mathbf{B}}(\tilde{T})$ is given by

$$
\tilde{\mathbf{B}}(\tilde{T})=\left((\tilde{\mathbf{H}}(\tilde{T}))^{H} \tilde{\mathbf{H}}(\tilde{T})+\lambda \mathbf{I}_{N_{T} M}\right)^{-1}(\tilde{\mathbf{H}}(\tilde{T}))^{H} .
$$

One should note that in the MIMO case, the matrix $\boldsymbol{\Lambda}^{(r)} \in$ $\mathbb{C}^{N_{R} M \times N_{T} M}$ defined in (6) is not diagonal. However, it has a $N_{R} \times N_{T}$ block structure where each submatrix $\Lambda_{i, j}^{(r)} \in$ $\mathbb{C}^{M \times M}$ is diagonal. Defining $\lambda_{m, i, j}^{(r)}$ as the $m$-th diagonal element of $\boldsymbol{\Lambda}_{i, j}^{(r)}$, we also define the matrix $\boldsymbol{\Lambda}_{m}^{(r)} \in \mathbb{C}^{N_{R} \times N_{T}}$ as

$$
\boldsymbol{\Lambda}_{m}^{(r)}=\left(\begin{array}{ccc}
\lambda_{m, 1,1}^{(r)} & \ldots & \lambda_{m, 1, N_{T}}^{(r)} \\
\vdots & \ddots & \vdots \\
\lambda_{m, N_{R}, 1}^{(r)} & \ldots & \lambda_{m, N_{R}, N_{T}}^{(r)}
\end{array}\right)
$$




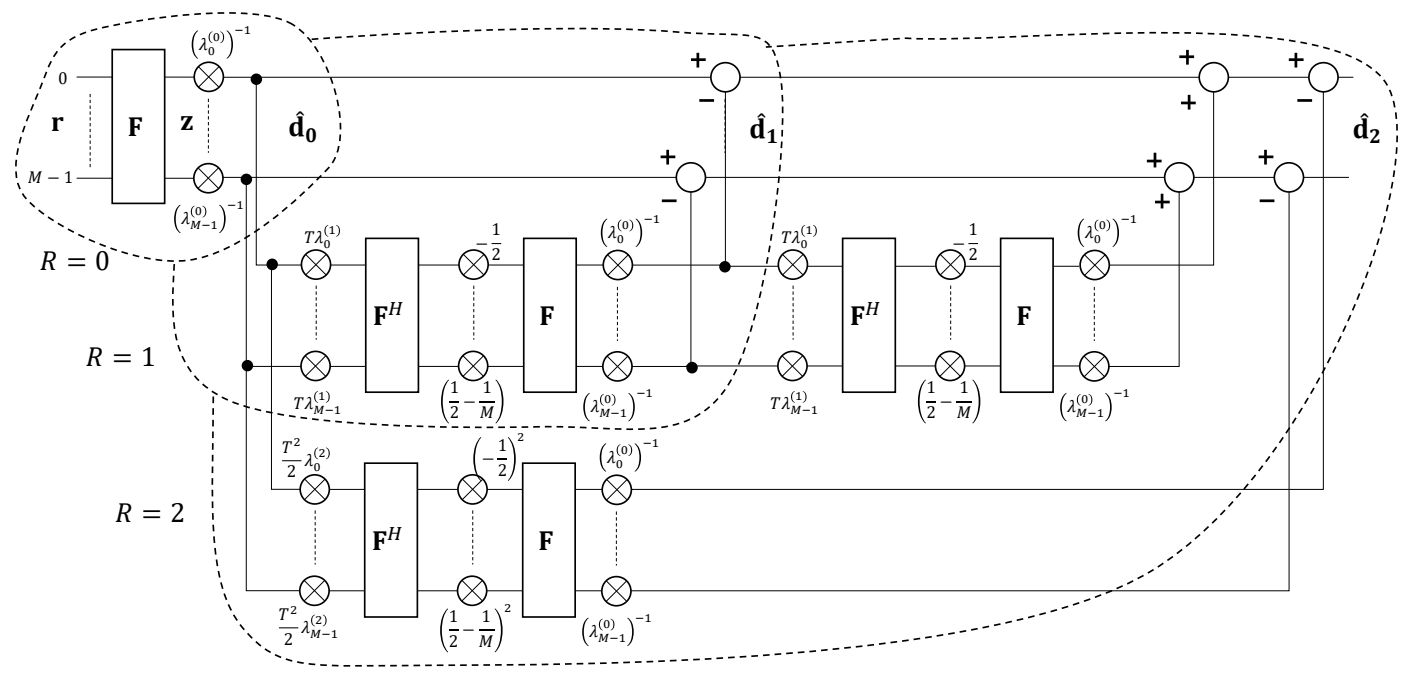

Fig. 1. Block diagram of the proposed equalizer in the SISO case $\left(N_{R}=N_{T}=1\right)$, with a zero forcing criterion and for $R=0$ (conventional OFDM receiver), $R=1$ and $R=2$. The scalar $\lambda_{m}^{(r)}$ corresponds to the $m$-th diagonal element of matrix $\Lambda^{(r)} \in \mathbb{C}^{M \times M}$.

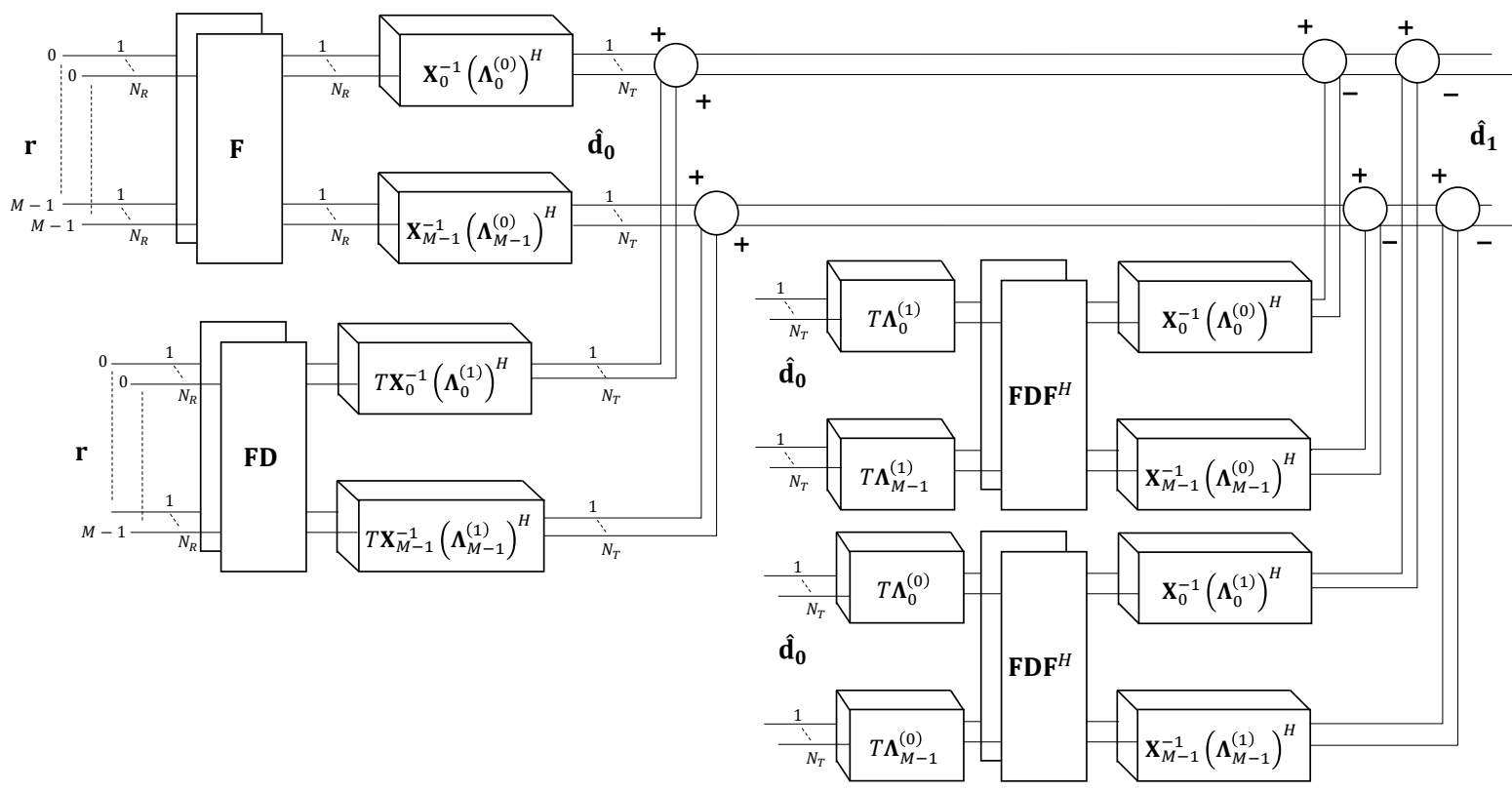

Fig. 2. Block diagram of the proposed equalizer in the MIMO case, with a MMSE criterion and for $R=0$ (conventional OFDM receiver) and $R=1$.

which corresponds to the $r$-th derivative with respect to time of the MIMO channel frequency response evaluated at subcarrier $m$. One can relate matrices $\boldsymbol{\Lambda}^{(r)}, \boldsymbol{\Lambda}_{i, j}^{(r)}$ and $\boldsymbol{\Lambda}_{m}^{(r)}$ as

$$
\begin{aligned}
\boldsymbol{\Lambda}^{(r)} & =\sum_{i=1}^{N_{R}} \sum_{j=1}^{N_{T}}\left(\mathbf{e}_{i, N_{R}} \mathbf{e}_{j, N_{T}}^{T} \otimes \boldsymbol{\Lambda}_{i, j}^{(r)}\right) \\
& =\sum_{m=0}^{M-1}\left(\boldsymbol{\Lambda}_{m}^{(r)} \otimes \mathbf{e}_{m, M} \mathbf{e}_{m, M}^{T}\right),
\end{aligned}
$$

where $\mathbf{e}_{n, N} \in \mathbb{R}^{N \times 1}$ is the $n$-th vector of the canonical basis. The expression (13) is very useful as it shows that a multiplication by the large matrix $\Lambda^{(r)}$ can be efficiently implemented on a subcarrier basis using $\Lambda_{m}^{(r)}$.
The zero order approximation of the equalizer is given by

$$
\begin{aligned}
\hat{\mathbf{B}}_{0} & =\left(\left(\mathbf{H}^{(0)}\right)^{H} \mathbf{H}^{(0)}+\lambda \mathbf{I}_{N_{T} M}\right)^{-1}\left(\mathbf{H}^{(0)}\right)^{H} \\
& =\left(\left(\boldsymbol{\Lambda}^{(0)}\right)^{H} \boldsymbol{\Lambda}^{(0)}+\lambda \mathbf{I}_{N_{T} M}\right)^{-1}\left(\boldsymbol{\Lambda}^{(0)}\right)^{H},
\end{aligned}
$$

which again corresponds to the conventional OFDM receiver with per-subcarrier MIMO channel equalization. Indeed, we can show that the large matrix inversion can actually be 
performed efficiently at the subcarrier level. Let us define

$$
\begin{aligned}
\mathbf{X} & =\left(\boldsymbol{\Lambda}^{(0)}\right)^{H} \boldsymbol{\Lambda}^{(0)}+\lambda \mathbf{I}_{N_{T} M} \\
\mathbf{X}_{m} & =\left(\boldsymbol{\Lambda}_{m}^{(0)}\right)^{H} \boldsymbol{\Lambda}_{m}^{(0)}+\lambda \mathbf{I}_{N_{T}} .
\end{aligned}
$$

Using (13) and the fact that $(\mathbf{A} \otimes \mathbf{B})=\mathbf{P}(\mathbf{B} \otimes \mathbf{A}) \mathbf{P}^{T}$ for square matrices $\mathbf{A}$ and $\mathbf{B}$ and a certain permutation matrix $\mathbf{P}$, we can write

$$
\begin{aligned}
\mathbf{X} & =\sum_{m=0}^{M-1}\left(\mathbf{X}_{m} \otimes \mathbf{e}_{m, M} \mathbf{e}_{m, M}^{T}\right) \\
& =\mathbf{P}\left(\sum_{m=0}^{M-1}\left(\mathbf{e}_{m, M} \mathbf{e}_{m, M}^{T} \otimes \mathbf{X}_{m}\right)\right) \mathbf{P}^{T} .
\end{aligned}
$$

One can note that the permuted matrix is block diagonal and hence, its inverse is given by another block diagonal matrix composed of the inverse of each block, i.e.,

$$
\begin{aligned}
\mathbf{X}^{-1} & =\mathbf{P}\left(\sum_{m=0}^{M-1}\left(\mathbf{e}_{m, M} \mathbf{e}_{m, M}^{T} \otimes \mathbf{X}_{m}^{-1}\right)\right) \mathbf{P}^{T} \\
& =\sum_{m=0}^{M-1}\left(\mathbf{X}_{m}^{-1} \otimes \mathbf{e}_{m, M} \mathbf{e}_{m, M}^{T}\right)
\end{aligned}
$$

The latter expression shows that the large matrix inversion $\mathbf{X}^{-1}$ can be efficiently computed and implemented at the subcarrier level using $\mathbf{X}_{m}^{-1}$. Let us define $\boldsymbol{\Psi}=\left(\mathbf{I}_{N_{R}} \otimes \mathbf{F D F}^{H}\right)$, which corresponds to $N_{R}$ parallel weighted FFT-IFFT pairs. Using Theorem 1 and the definition in (11), the first order approximation of the ideal receiver is given by

$$
\begin{aligned}
\hat{\mathbf{B}}_{1} & =\hat{\mathbf{B}}_{0}+\mathbf{X}^{-1}\left(\mathbf{H}^{(1)}\right)^{H}-\mathbf{X}^{-1} \\
& \left(\left(\mathbf{H}^{(1)}\right)^{H} \mathbf{H}^{(0)}+\left(\mathbf{H}^{(0)}\right)^{H} \mathbf{H}^{(1)}\right) \mathbf{X}^{-1}\left(\mathbf{H}^{(0)}\right)^{H} \\
& =\hat{\mathbf{B}}_{0}+\mathbf{X}^{-1}\left(\boldsymbol{\Lambda}^{(1)}\right)^{H} \boldsymbol{\Psi}-\mathbf{X}^{-1} \\
& \left(\left(\boldsymbol{\Lambda}^{(1)}\right)^{H} \boldsymbol{\Psi} \boldsymbol{\Lambda}^{(0)}+\left(\boldsymbol{\Lambda}^{(0)}\right)^{H} \boldsymbol{\Psi} \boldsymbol{\Lambda}^{(1)}\right) \mathbf{X}^{-1}\left(\boldsymbol{\Lambda}^{(0)}\right)^{H} .
\end{aligned}
$$

The block diagram of this equalizer is depicted in Fig. 2. As in the SISO case, the equalizer structure only consists in persubcarrier multiplications and weighted-FFT-IFFT pairs.

\section{Complexity Analysis}

Two types of complexity for an equalizer can be distinguished: 1) the complexity of computing its coefficients and 2) the complexity of its implementation.

1) Coefficients computation: The main advantage of the proposed equalizer is that the complexity of computing its coefficients is similar to that of the conventional single-tap OFDM receiver. Indeed, looking at Fig. 1 and Fig. 2, we can see that the proposed equalizers use the same channel depending quantities as conventional OFDM: the channel frequency response at each subcarrier $\Lambda_{m}^{(0)}$ and the regularized inverse of its Gram matrix $\mathbf{X}_{m}^{-1}$.

The proposed equalizer only requires the additional computation of the time derivatives of the channel frequency response
$\boldsymbol{\Lambda}_{m}^{(r)}$ at each subcarrier $m$ and for $0<r \leq R$. In this paper, we assumed that the receiver has perfect knowledge of the channel. In practice, this is of course not the case and the channel frequency response and its derivatives need to be estimated based on pilots scattered in time and frequency. Different options are possible to estimate the derivatives $\boldsymbol{\Lambda}_{m}^{(r)}$ for $0<r \leq R$. A first option is to estimate them by finite difference between channel estimates $\boldsymbol{\Lambda}_{m}^{(0)}$ at successive OFDM symbols. Another option is to estimate the Doppler support of the channel and derive the time derivatives by computing an inverse Fourier transform. Note that, due to the time selectivity, pilot tones might suffer from ICI and proper schemes need to be used to avoid this issue [21], [22].

2) Implementation complexity: On the other hand, the complexity of implementation of the proposed equalizers obviously requires more operations than conventional single-tap OFDM receivers. However, the additional operations only consist of FFT's and IFFT's and per-subcarrier matrix multiplications, which result in a low additional complexity burden. Table I shows the complexity of implementation of the proposed equalizer in terms of real-valued multiplications. For the calculation, we assumed that the number of subcarriers is a power of two and that an FFT/IFFT of size $M$ requires $M\left(\log _{2}(M)-3\right)+4$ real-valued multiplications using the split-radix algorithm [23].

In the ZF $N_{R}=N_{T}$ case, the channel matrix is square and this can leveraged to decrease the implementation complexity of the equalizer. For the $R=1$ and $R=2$, it respectively requires around three and seven times more realvalued multiplications than for the conventional single-tap OFDM equalizer. For the MMSE equalizer or the ZF equalizer with $N_{R}>N_{T}$, the equalizer is around six times more complex to implement than the conventional one for $R=1$ and around 17 times more complex for $R=2$. We will show in the simulation section that the $R=1$ order of the proposed equalizer is already very efficient to compensate for the distortion implying that increasing the order $R$ might not be necessary in conventional applications.

Note that the low implementation complexity of the presented equalizer is not achieved by most of the proposed solutions in the literature. As a comparison, in the SISO case, [13] would require $O\left(M^{2}\right)$ multiplications to compute the channel matrix and perform interference cancellation. On the other hand, the serial and block equalizers studied in [2], relying on a banded approximation of the channel matrix $\mathbf{H}$ and using $L D L^{H}$ factorization, would require $O\left(M Q^{2}\right)$ multiplications with $Q$ being the upper and lower bandwidth.

\section{Performance Analysis}

We are interested in a practical expression for computing the performance of the proposed equalizer. This could for instance help the receiver to fix the value of $R$ as a function of a performance target. In the following, we assume that the entries of the symbol vector $\mathbf{d}$ are i.i.d. random variables with zero mean and variance $E_{S} / N_{T}$. 
TABLE I

IMPLEMENTATION COMPLEXITY OF THE EQUALIZER IN TERMS OF REAL-VALUED MULTIPLICATIONS.

\begin{tabular}{|c|c|c|}
\hline & Zero forcing for $N_{T}=N_{R}$ & MMSE and zero forcing for $N_{R}>N_{T}$ \\
\hline Conventional single-tap equalizer $(R=0)$ & $N_{R} M\left(\log _{2}(M)-3+4 N_{R}\right)+4 N_{R}$ & $N_{R} M\left(\log _{2}(M)-3+4 N_{T}\right)+4 N_{R}$ \\
\hline Proposed equalizer, $R=1$ & $N_{R} M\left(3 \log _{2}(M)-7+12 N_{R}\right)+12 N_{R}$ & $N_{R} M\left(6 \log _{2}(M)-12+24 N_{T}\right)+24 N_{R}$ \\
\hline Proposed equalizer, $R=2$ & $N_{R} M\left(7 \log _{2}(M)-15+28 N_{R}\right)+28 N_{R}$ & $N_{R} M\left(17 \log _{2}(M)-33+76 N_{T}\right)+68 N_{R}$ \\
\hline
\end{tabular}

We now also consider the effect of additive noise that we neglected so far. The equalized symbols are then given by

$$
\hat{\mathbf{d}}_{R}=\hat{\mathbf{B}}_{R} \mathbf{H d}+\hat{\mathbf{B}}_{R}\left(\mathbf{I}_{N_{R}} \otimes \mathbf{F}\right) \mathbf{w},
$$

where the vector $\mathbf{w} \in \mathbb{C}^{N_{R} M \times 1}$ contains the noise samples. The figure of merit that we study is the NMSE of the equalized symbols, which correlation matrix is given by

$$
\mathbf{E}_{R}=\frac{\mathbb{E}\left(\left(\hat{\mathbf{d}}_{R}-\mathbf{d}\right)\left(\hat{\mathbf{d}}_{R}-\mathbf{d}\right)^{H}\right)}{E_{S} / N_{T}},
$$

where the expectation is taken over the transmitted symbols and the additive noise samples, for one specific channel realization $\mathbf{C}$. Note that $\mathbf{E}_{R}$ is a $N_{T} M \times N_{T} M$ correlation matrix. The variance of the NMSE associated to the $k$-th stream and to the $m$-th subcarrier is given by its $m+k M$ diagonal entry for $k=0, \ldots, N_{T}-1$ while its trace gives the total aggregated NMSE.

We assume that the noise is not correlated with the data symbols. If the received signal at each antenna is impacted by additive circularly-symmetric white Gaussian noise, with zero mean and variance $N_{0}$, we find that

$$
\begin{aligned}
\mathbf{E}_{R} & =\left(\hat{\mathbf{B}}_{R} \mathbf{H}-\mathbf{I}_{N_{T} M}\right)\left(\hat{\mathbf{B}}_{R} \mathbf{H}-\mathbf{I}_{N_{T} M}\right)^{H} \\
& +\frac{N_{T} N_{0}}{E_{S}} \hat{\mathbf{B}}_{R} \hat{\mathbf{B}}_{R}^{H} .
\end{aligned}
$$

The additive noise contribution is easy to evaluate as the equalizer $\hat{\mathbf{B}}_{R}$ is composed of low complexity operations such as FFT, IFFT and per-subcarrier multiplications. On the other hand, the distortion term $\mathbf{E}_{R, d}$, defined as

$$
\mathbf{E}_{R, d}=\left(\hat{\mathbf{B}}_{R} \mathbf{H}-\mathbf{I}_{N_{T} M}\right)\left(\hat{\mathbf{B}}_{R} \mathbf{H}-\mathbf{I}_{N_{T} M}\right)^{H},
$$

is composed of inter-carrier and inter-antenna interference and results from the fact that the channel is not perfectly inverted. This term is more complex to evaluate as it requires to perform the multiplication of large matrices and no a priori known structure of matrix $\mathbf{H}$ that can be exploited to simplify the computation. Therefore, we propose in the following an approximation of the distortion term $\mathbf{E}_{R, d}$, which can be efficiently computed and will prove very accurate in practical situations.

\section{A. General Expression of the Distortion}

Quite naturally, we define the $R$-th order approximation of $\mathbf{H}$ as the $R$-th order Taylor expansion of $\tilde{\mathbf{H}}(\tilde{T})$ around $\tilde{T}=0$ and evaluated at $\tilde{T}=T$ giving

$$
\hat{\mathbf{H}}_{R}=\sum_{r=0}^{R} \frac{T^{r}}{r !}\left(\mathbf{I}_{N_{R}} \otimes \mathbf{F} \mathbf{D}^{r} \mathbf{F}^{H}\right) \boldsymbol{\Lambda}^{(r)} .
$$

We also define the following approximation of $\mathbf{B}_{R} \mathbf{H}$

$$
\hat{\mathbf{\Psi}}_{R}=\sum_{r=0}^{R} \frac{T^{r}}{r !} \mathbf{B}^{(r)} \hat{\mathbf{H}}_{R+1-r} .
$$

Theorem 2. An approximation of $\mathbf{E}_{r, d}$ is given by

$$
\hat{\mathbf{E}}_{R, d}=\left(\hat{\mathbf{\Psi}}_{R}-\mathbf{I}_{N_{T} M}\right)\left(\hat{\mathbf{\Psi}}_{R}-\mathbf{I}_{N_{T} M}\right)^{H},
$$

and, under (As1) $-(\mathbf{A s 2})$ and as $T \rightarrow 0$, the error is bounded as

$$
\left\|\mathbf{E}_{R, d}-\hat{\mathbf{E}}_{R, d}\right\|=O\left(T^{R+1}\right) .
$$

Proof. The proof is given in Appendix VII-C.

Theorem 2 gives a practical expression for evaluating the performance of the system. The main benefit of using $\hat{\mathbf{\Psi}}_{R}$ is that it only consists of basic operations such as per-subcarrier multiplications and FFT-IFFT pairs, which allows for low complexity computations. In the simulation section, we will see that the approximation $\hat{\mathbf{E}}_{R, d}$ is very close to the actual performance metric $\mathbf{E}_{R, d}$.

\section{B. Zero Forcing Case}

We now introduce additional assumptions, required by Corollary 1.

(As2b): The equalizer function $\tilde{\mathbf{B}}(\tilde{T})$ is $C^{R+2}$ smooth for $\tilde{T} \in[0, T]$.

(As3): The equalizer function $\tilde{\mathbf{B}}(\tilde{T})$ inverts the end-to-end transfer function $\tilde{\mathbf{H}}(\tilde{T})$ for $\tilde{T} \in[0, T]$, i.e.,

$$
\tilde{\mathbf{B}}(\tilde{T}) \tilde{\mathbf{H}}(\tilde{T})=\mathbf{I}_{N_{T} M} \text { for } \tilde{T} \in[0, T] .
$$

The following theorem gives a compact approximation for the distortion $\mathbf{E}_{R, d}$ in the case where the ideal equalizer function $\tilde{\mathbf{B}}(\tilde{T})$ inverts the channel at the receiver (As4).

Corollary 1. Under (As1), (As2b) and (As3), the expression of $\hat{\mathbf{E}}_{R, d}$ proposed in Theorem 2 simplifies to

$$
\hat{\mathbf{E}}_{R, d}=\frac{T^{2 R+2}}{((R+1) !)^{2}} \mathbf{B}^{(R+1)} \boldsymbol{\Lambda}^{(0)}\left(\mathbf{B}^{(R+1)} \boldsymbol{\Lambda}^{(0)}\right)^{H},
$$

and, as $T \rightarrow 0$, the error is bounded as

$$
\left\|\mathbf{E}_{R, d}-\hat{\mathbf{E}}_{R, d}\right\|=O\left(T^{2 R+2}\right) .
$$

Proof. The proof is given in Appendix VII-D.

The corollary shows that, under (As3), many terms related to the fact that the channel is not completely inverted are canceled, which greatly simplifies the expression of $\hat{\mathbf{E}}_{R, d}$. Note that, for instance, $\hat{\mathbf{E}}_{0, d}$ may give the expression of the performance of the conventional OFDM receiver $(R=0)$. 
Indeed, the expression for the performance of a SISO zero forcing equalizer, as considered in Section III-B1, is ${ }^{1}$

$$
\begin{aligned}
& \hat{\mathbf{E}}_{0, d}=T^{2} \mathbf{B}^{(1)} \boldsymbol{\Lambda}^{(0)}\left(\boldsymbol{\Lambda}^{(0)}\right)^{H}\left(\mathbf{B}^{(1)}\right)^{H} \\
& =T^{2}\left(\boldsymbol{\Lambda}^{(0)}\right)^{-1} \mathbf{F D F} \mathbf{\Lambda}^{H} \boldsymbol{\Lambda}^{(1)}\left(\boldsymbol{\Lambda}^{(1)}\right)^{H} \mathbf{F D F}^{H}\left(\boldsymbol{\Lambda}^{(0)}\right)^{-H} .
\end{aligned}
$$

\section{Simulation Results}

This section aims at demonstrating the performance of the proposed designs and approximations through simulations. Most results of this paper are derived using an asymptotic argument, meaning that they become true as the OFDM symbol period $T$ goes to zero. We will show in the following that, even for highly time-varying channels, the symbol period $T$ can be considered low enough so that the approximations are valid and match the simulation results. To quantify the amount of time selectivity relatively to the symbol period, we will use the product $f_{d} T$ in the following simulations.

We consider an OFDM system with $M=512$ subcarriers and a channel length $L=\frac{M}{8}=64$. As previously explained, the parameter $T$ is the fundamental parameter related to the system robustness to channel time variations. In this sense, varying the number of subcarriers $M$ does not impact the system robustness as long as the product $f_{d} T$ remains constant. We assume that the cyclic prefix length is longer than $L$. Each channel tap $\phi_{i, j, l}(\nu)$ follows a Rayleigh distribution with an exponentially decaying power delay profile. We assume no spatial correlation between antennas. We consider two types of time-varying channels: either each tap has a Jakes Doppler spectrum (3) or the taps are static in time but each transmit antenna is impacted by a different carrier frequency offset, which models a typical MU MIMO scenario.

\section{A. SISO Scenario}

1) Performance of the proposed equalizer and accuracy of the NMSE approximation for one channel realization: Let us consider the SISO case first. In Fig. 3, we consider one channel realization. For this realization, we plotted the NMSE at one OFDM multicarrier symbol as a function of the subcarrier index for a SNR $\frac{E_{S}}{N_{0}}=25 \mathrm{~dB}$ and a product $f_{d} T=0.1$. We used a high SNR regime to highlight the gain of the proposed equalizers. Indeed, at low SNR's, the distortion power becomes negligible compared to the noise power. Different versions of the proposed equalizers are plotted: $R=1$ and $R=2$. The conventional OFDM implementation corresponds to single-tap per-subcarrier equalization $(R=0)$ and the ideal equalizer is given in (9). A zero forcing is here considered, i.e., $\lambda=0$. The proposed equalizers $R=1$ and $R=2$ are compared to the state of the art "block banded equalizer", which is obtained by approximating the ideal equalizer in (9) as

$$
\mathbf{B}_{\text {Block banded }}=\left(\mathbf{H}_{\text {Banded }}^{H} \mathbf{H}_{\text {Banded }}+\lambda \mathbf{I}_{M}\right)^{-1} \mathbf{H}_{\text {Banded }}^{H},
$$

\footnotetext{
${ }^{1}$ To get the full NMSE expression, one should still add the additive noise contribution given by $\frac{E_{S}}{N_{0}} \hat{\mathbf{B}}_{0} \hat{\mathbf{B}}_{0}^{H}$.
}

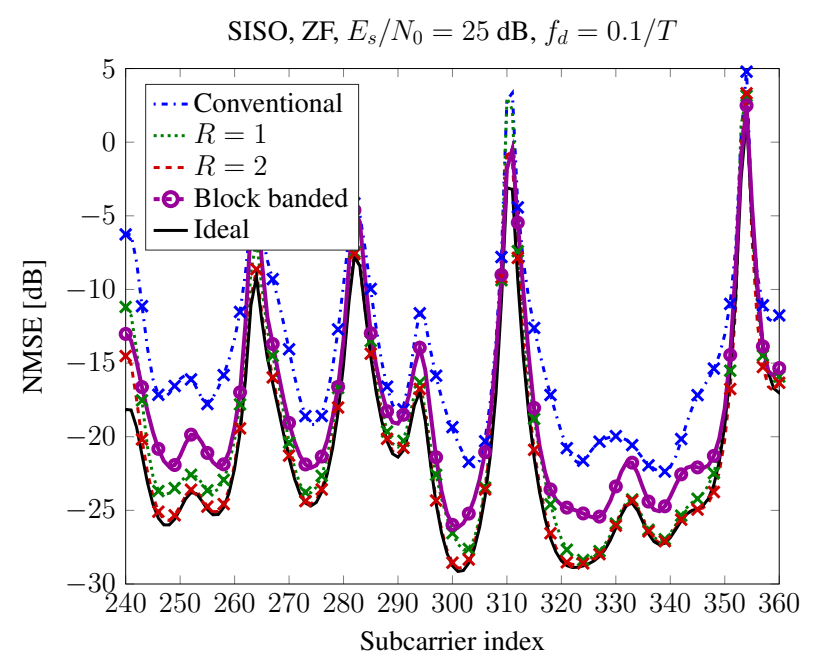

Fig. 3. The proposed equalizers allow to get close to the ideal equalizer as $R$ increases. The simulated performance of the proposed receivers, plotted with crosses, closely matches the lines that represent the proposed approximation of the NMSE in Section IV.

where the band matrix $\mathbf{H}_{\text {Banded }}$ is obtained by selecting the main diagonal, the $Q$ superdiagonals and $Q$ subdiagonals of $\mathbf{H}$, including its upper right and lower left elements. We chose the value $Q=2$ as in [2]. We chose to compare to this type of equalizer as it is a well known reference in the literature and provides a good trade-off between performance and complexity of implementation.

One can first see a clear gain of the proposed equalizers versus both the conventional receiver and the block banded equalizer. The gain becomes larger as $R$ increases and gets close to the ideal equalizer curve. Furthermore, the simulated performance of the proposed receivers, plotted with crosses, matches the lines that represent the proposed approximation of the NMSE in Section IV, which imply that the proposed expressions are very accurate in practice. For the sake of clarity of the following figures, we will only plot the simulated performance of the proposed equalizers.

2) Cumulative density function of the NMSE: In Fig. 4, the cumulative density function of the NMSE at each subcarrier is evaluated over 100 channel realizations, for an MMSE type of receiver and different values of the ratio $\frac{E_{S}}{N_{0}}$. One can see that the proposed design outperforms the block banded equalizer for more than $90 \%$ of channel realizations. In the top region of the graph, their performance converge for low values of the NMSE.

\section{B. MIMO Scenario}

Let us now consider a MIMO scenario with $N_{R}=3$ and $N_{T}=2$ and a MMSE receiver. Fig. 5 shows the cumulative density function of the NMSE evaluated at each subcarrier and spatial stream and estimated over 100 channel realizations for two $\frac{E_{S}}{N_{0}}$ ratios. Again, the same conclusions as in the SISO case hold and the proposed equalizers outperform the MIMO block banded equalizer which was defined as

$\mathbf{B}_{\text {Block banded }}^{\text {MIMO }}=\left(\mathbf{H}_{\text {Banded }}^{H} \mathbf{H}_{\text {Banded }}+\lambda \mathbf{I}_{N_{T} M}\right)^{-1} \mathbf{H}_{\text {Banded }}^{H}$, 
SISO, LMMSE, $f_{d}=0.1 / T$

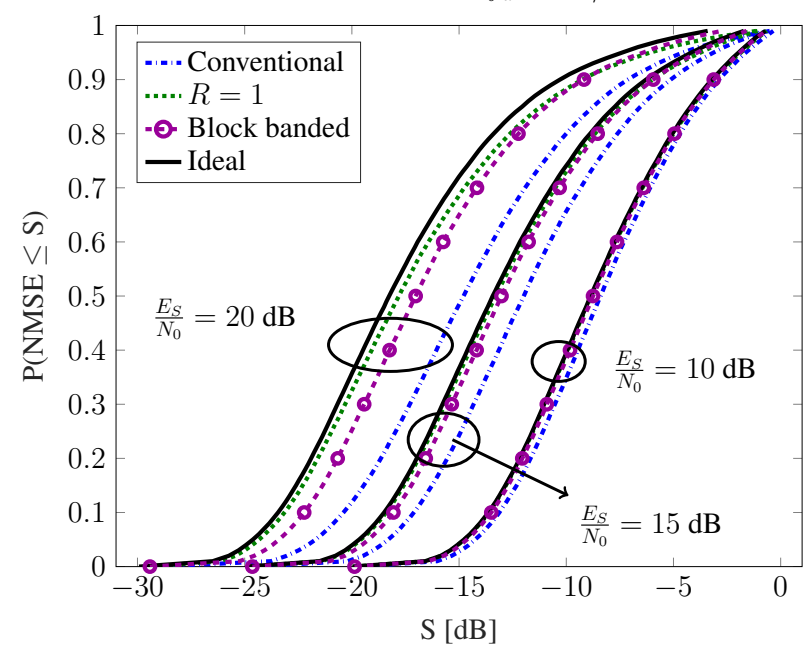

Fig. 4. Cumulative density function of the NMSE evaluated at each subcarrier estimated with 100 channel realizations. The proposed equalizer $R=1$ outperforms a block banded equalizer with semi-bandwidth $Q=2$.

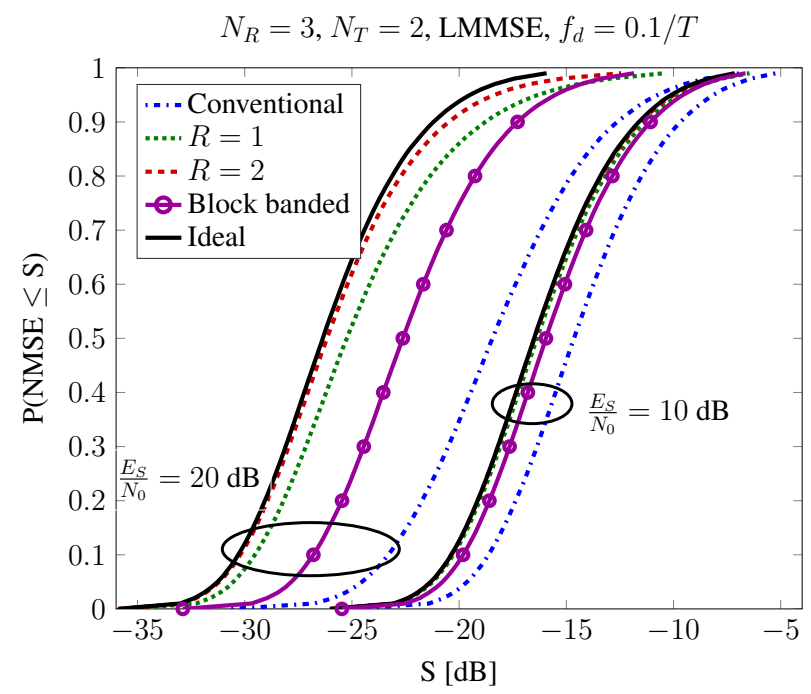

Fig. 5. Cumulative density function of the NMSE evaluated at each subcarrier estimated with 100 channel realizations in the MIMO case. The proposed equalizer outperforms the block banded equalizer with semi-bandwidth $Q=$ 2 .

where the matrix $\mathbf{H}_{\text {Banded }} \in \mathbb{C}^{N_{R} M \times N_{T} M}$ can be decomposed in $N_{R} \times N_{T}$ submatrices of size $M \times M$. Each of these submatrices is approximated as a banded matrix by selecting from the corresponding submatrix of $\mathbf{H}$ its main diagonal, the $Q$ superdiagonals and $Q$ subdiagonals, including its upper right and lower left elements. We chose here again the value $Q=2$.

\section{Multi-user MIMO Scenario}

Fig. 6 addresses an uplink MU MIMO scenarios. Each transmit antenna is affected by a different carrier frequency offset uniformly drawn between $-0.25 / T$ and $0.25 / T$. We can see that the conventional OFDM receiver saturates as the SNR increases due to the inter-carrier interference induced by

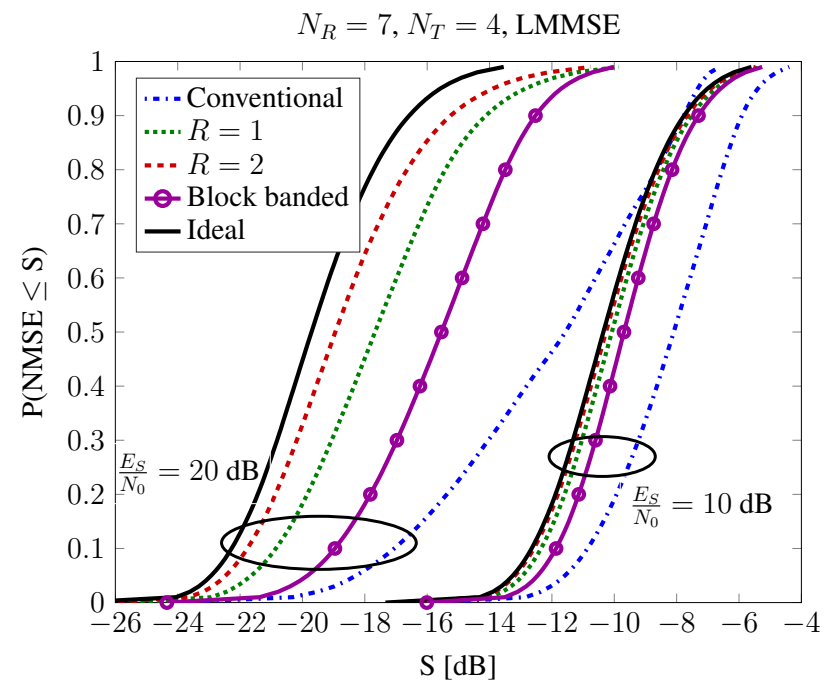

Fig. 6. Cumulative density function of the NMSE evaluated at each subcarrier estimated with 100 channel realizations in the multi-user uplink MIMO case. For each realization, a different $\mathrm{CFO}$ offset per-user is drawn from a uniform distribution between $-0.25 / T$ and $0.25 / T$.

the carrier frequency offsets. On the other hand, the proposed equalization structures are very efficient to compensate for the carrier frequency offset and outperform the MIMO block banded equalizer.

\section{CONCLUSions}

We investigated the compensation of time-varying channels in MIMO OFDM systems. We proposed a novel equalization structure obtained by Taylor approximation of the ideal channel equalizer function. The obtained approximations were shown to result in very efficient implementation. On the one hand, the complexity of computing the equalizer coefficients only require the knowledge of the derivatives of the channel frequency response. On the other hand, the complexity of implementing the equalizer was shown to be very low as the equalizer can be efficiently implemented through persubcarrier processing and FFT/IFFT operations. The performance of the proposed equalizers was both analytically and numerically validated, which showed that they are extremely efficient to compensate for time-varying channels or multiple CFOs.

\section{APPENDIX}

\section{A. Proof of Proposition 1}

Under (As1), using the fact that the convergence radius of the exponential function is infinite, we can obtain that the function $\tilde{c}_{i, j, l}(t)$ is analytic. Hence, its Taylor expansion converges for any $t$. Let us express $\tilde{c}_{i, j, l}(t)$ as its Taylor 
expansion around the time instant $t=0$ that we evaluate at $t_{n}=\left(n-\frac{M}{2}\right) \frac{T}{M}$, which gives

$$
\begin{aligned}
c_{i, j}[l, n] & =\tilde{c}_{i, j, l}\left(t_{n}\right) \\
& =\sum_{r=0}^{+\infty} \frac{\left.\frac{d^{(r)}}{d t^{r}} \tilde{c}_{i, j, l}(t)\right|_{t=0}}{r !} t_{n}^{r} \\
& =\sum_{r=0}^{+\infty} \frac{T^{r}}{r !} c_{i, j}^{(r)}[l]\left(\frac{n-\frac{M}{2}}{M}\right)^{r},
\end{aligned}
$$

where $c_{i, j}^{(r)}[l]$ was defined in (4). We can thus rewrite matrix $\mathbf{C}_{i, j}$, which structure is shown in (1), as

$$
\mathbf{C}_{i, j}=\sum_{r=0}^{+\infty} \frac{T^{r}}{r !} \mathbf{D}^{r} \mathbf{C}_{i, j}^{(r)}
$$

and

$$
\mathbf{C}=\sum_{r=0}^{+\infty} \frac{T^{r}}{r !}\left(\mathbf{I}_{N_{R}} \otimes \mathbf{D}^{r}\right) \mathbf{C}^{(r)}
$$

where matrices $\mathbf{D}, \mathbf{C}_{i, j}$ and $\mathbf{C}^{(r)}$ were defined in Proposition 1. Note that this derivation could have been done for an arbitrary $T$. To avoid ambiguity, we define the function $\tilde{\mathbf{C}}(\tilde{T})$ valid for any $\tilde{T}$ and such that $\mathbf{C}=\tilde{\mathbf{C}}(T)$, as defined in Proposition 1. This completes the proof.

\section{B. Proof of Theorem 1}

The equalizer $\hat{\mathbf{B}}_{R}$ is obtained as the Taylor expansion of $\tilde{\mathbf{B}}(\tilde{T})$ around $T=0$, evaluated at $\tilde{T}=T$ and truncated to its $R+1$ first order terms. Using the Lagrange expression of the remainder of a Taylor expansion [24], we can write that, for some $\hat{T} \in[0, T]$, we have

$$
\mathbf{B}-\hat{\mathbf{B}}_{R}=\left.\frac{T^{R+1}}{(R+1) !} \frac{d^{(R+1)}}{d \tilde{T}^{R+1}} \tilde{\mathbf{B}}(\tilde{T})\right|_{\tilde{T}=\hat{T}} .
$$

Taking the norm and using the fact that, under (As2), the $R+1$-th derivative of $\tilde{\mathbf{B}}(\tilde{T})$ is bounded, we find the result of Theorem 1.

\section{Proof of Theorem 2}

We begin by proving Lemmas 1 and 2 that will prove useful to demonstrate Theorem 2.

Lemma 1. Under (As1) and as $T \rightarrow 0$, we have

$$
\left\|\mathbf{H}-\hat{\mathbf{H}}_{R}\right\|=O\left(T^{R}\right)
$$

where $\hat{\mathbf{H}}_{R}$ is defined in (15).

Proof. Using (8), we can write

$$
\begin{aligned}
\left\|\mathbf{H}-\hat{\mathbf{H}}_{R}\right\| & =\left\|\sum_{r=R+1}^{+\infty} \frac{T^{r}}{r !}\left(\mathbf{I}_{N_{R}} \otimes \mathbf{F} \mathbf{D}^{r} \mathbf{F}^{H}\right) \mathbf{\Lambda}^{(r)}\right\| \\
& \leq \sum_{r=R+1}^{+\infty} \frac{T^{r}}{r !}\left\|\left(\mathbf{I}_{N_{R}} \otimes \mathbf{F} \mathbf{D}^{r} \mathbf{F}^{H}\right) \mathbf{\Lambda}^{(r)}\right\|,
\end{aligned}
$$

where we used the triangular inequality to get the sum out of the norm. Using the identity $\|\mathbf{A B}\| \leq \lambda_{\max }^{1 / 2}\left(\mathbf{A A}^{H}\right)\|\mathbf{B}\|$, we can further write

$$
\begin{aligned}
\left\|\mathbf{H}-\hat{\mathbf{H}}_{R}\right\| & \leq \sum_{r=R+1}^{+\infty} \frac{T^{r}}{r !} \lambda_{\max }^{1 / 2}\left(\mathbf{D}^{2 r}\right)\left\|\boldsymbol{\Lambda}^{(r)}\right\| \\
& \leq \sum_{r=R+1}^{+\infty} \frac{T^{r}}{r !}\left(\frac{1}{2}\right)^{r}\left\|\boldsymbol{\Lambda}^{(r)} .\right\|
\end{aligned}
$$

Now, using the expression in (13) for $\Lambda^{(r)}$ and the triangular inequality, we can write

$$
\left\|\boldsymbol{\Lambda}^{(r)}\right\| \leq \sum_{m=0}^{M-1}\left\|\boldsymbol{\Lambda}_{m}^{(r)} \otimes \mathbf{e}_{m, M} \mathbf{e}_{m, M}^{T}\right\| \leq \sum_{m=0}^{M-1}\left\|\boldsymbol{\Lambda}_{m}^{(r)}\right\| .
$$

Now, let us define matrices $\mathbf{C}^{(r)}[l] \in \mathbb{C}^{N_{R} \times N_{T}}$ and $\boldsymbol{\Phi}_{l}(\nu) \in$ $\mathbb{C}^{N_{R} \times N_{T}}$ as

$$
\begin{aligned}
\mathbf{C}^{(r)}[l] & =\left(\begin{array}{ccc}
c_{1,1}^{(r)}[l] & \ldots & c_{1, N_{T}}^{(r)}[l] \\
\vdots & \ddots & \vdots \\
c_{N_{R}, 1}^{(r)}[l] & \ldots & c_{N_{R}, N_{T}}^{(r)}[l]
\end{array}\right) \\
\boldsymbol{\Phi}_{l}(\nu) & =\left(\begin{array}{ccc}
\phi_{1,1, l}(\nu) & \ldots & \phi_{1, N_{T}, l}(\nu) \\
\vdots & \ddots & \vdots \\
\phi_{N_{R}, 1, l}(\nu) & \ldots & \phi_{N_{R}, N_{T}, l}(\nu)
\end{array}\right),
\end{aligned}
$$

and under (As1), these matrices are related as

$$
\mathbf{C}^{(r)}[l]=\int_{-f_{d}}^{f_{d}}(\jmath 2 \pi \nu)^{r} \boldsymbol{\Phi}_{l}(\nu) d \nu
$$

and related to $\boldsymbol{\Lambda}_{m}^{(r)}$ through

$$
\boldsymbol{\Lambda}_{m}^{(r)}=\sum_{l=0}^{L-1} \mathbf{C}^{(r)}[l] e^{-\jmath \frac{2 \pi}{M} l m} .
$$

Coming back to (18), using the above definitions and the triangular inequality, we can write

$$
\begin{aligned}
\left\|\boldsymbol{\Lambda}^{(r)}\right\| & \leq \sum_{m=0}^{M-1} \sum_{l=0}^{L-1}\left\|\mathbf{C}^{(r)}[l]\right\| \\
& \leq M\left(2 \pi f_{d}\right)^{r} \sum_{l=0}^{L-1} \int_{-f_{d}}^{f_{d}}\left\|\mathbf{\Phi}_{l}(\nu)\right\| d \nu .
\end{aligned}
$$

We define $\xi=\sum_{l=0}^{L-1} \int_{-f_{d}}^{f_{d}}\left\|\boldsymbol{\Phi}_{l}(\nu)\right\| d \nu$, which is bounded as the channel has a finite energy. Replacing in (17), we finally find that

$$
\begin{aligned}
\left\|\mathbf{H}-\hat{\mathbf{H}}_{R}\right\| & \leq \xi M \sum_{r=R+1}^{+\infty} \frac{\left(\pi f_{d} T\right)^{r}}{r !} \\
& =O\left(T^{R}\right) .
\end{aligned}
$$

This concludes the proof of Lemma 1.

Lemma 2. Under (As1)-(As2) and as $T \rightarrow 0$, we have

$$
\left\|\hat{\mathbf{B}}_{R} \mathbf{H}-\hat{\mathbf{\Psi}}_{R}\right\|=O\left(T^{R+1}\right),
$$

where $\hat{\mathbf{\Psi}}_{R}$ is defined in (16). 
Proof. Using the definition of $\hat{\mathbf{B}}_{R}$ in Theorem 1 and the result of Lemma 1, we can write

$$
\begin{aligned}
\hat{\mathbf{B}}_{R} \mathbf{H} & =\sum_{r=0}^{R} \frac{T^{r}}{r !} \mathbf{B}^{(r)} \mathbf{H} \\
& =\sum_{r=0}^{R} \frac{T^{r}}{r !} \mathbf{B}^{(r)}\left(\hat{\mathbf{H}}_{R+1-r}+O\left(T^{R+1-r}\right)\right),
\end{aligned}
$$

as $T \rightarrow 0$. Identifying the expression of $\hat{\mathbf{\Psi}}_{R}$, we conclude that

$$
\left\|\hat{\mathbf{B}}_{R} \mathbf{H}-\hat{\mathbf{\Psi}}_{R}\right\|=O\left(T^{R+1}\right) \sum_{r=0}^{R} \frac{\mathbf{B}^{(r)}}{r !}=O\left(T^{R+1}\right),
$$

using the fact that, under (As2), the derivatives of the equalizer are bounded.

We are now in the position of demonstrating Theorem 2. Let us define the error matrix $\boldsymbol{\Theta}_{R}=\hat{\mathbf{B}}_{R} \mathbf{H}-\hat{\mathbf{\Psi}}_{R}$. We have

$$
\begin{aligned}
\mathbf{E}_{R, d} & =\left(\hat{\mathbf{\Psi}}_{R}+\boldsymbol{\Theta}_{R}-\mathbf{I}_{N_{T} M}\right)\left(\hat{\mathbf{\Psi}}_{R}+\boldsymbol{\Theta}_{R}-\mathbf{I}_{N_{T} M}\right)^{H} \\
& =\hat{\mathbf{E}}_{R, d}+\boldsymbol{\Theta}_{R} \boldsymbol{\Theta}_{R}^{H}+\boldsymbol{\Theta}_{R}\left(\hat{\mathbf{\Psi}}_{R}-\mathbf{I}_{N_{T} M}\right)^{H} \\
& +\left(\hat{\mathbf{\Psi}}_{R}-\mathbf{I}_{N_{T} M}\right) \boldsymbol{\Theta}_{R}^{H} .
\end{aligned}
$$

Using Lemma 2, we have that, as $T \rightarrow 0,\left\|\boldsymbol{\Theta}_{R}\right\|=O\left(T^{R+1}\right)$. Under (As1) and (As2), matrix $\left(\hat{\mathbf{\Psi}}_{R}-\mathbf{I}_{N_{T} M}\right)$ is bounded. Hence, we can conclude that

$$
\left\|\mathbf{E}_{R, d}-\hat{\mathbf{E}}_{R, d}\right\|=O\left(T^{R+1}\right) .
$$

\section{Proof of Corollary 1}

First, note that, under (As3), we have $\tilde{\mathbf{B}}(\tilde{T}) \tilde{\mathbf{H}}(\tilde{T})=$ $\mathbf{I}_{N_{T} M}$, which does not depend on the auxiliary symbol period $\tilde{T}$ and hence

$$
\begin{aligned}
\left.\frac{d^{(r)}}{d \tilde{T}^{r}}(\tilde{\mathbf{B}}(\tilde{T}) \tilde{\mathbf{H}}(\tilde{T}))\right|_{\tilde{T}=0} & =\sum_{r^{\prime}=0}^{r} \frac{r !}{r^{\prime} !\left(r-r^{\prime}\right) !} \mathbf{B}^{\left(r^{\prime}\right)} \mathbf{H}^{\left(r-r^{\prime}\right)} \\
& =\delta_{r} \mathbf{I}_{N_{T} M} .
\end{aligned}
$$

Now, let us use the definition of $\hat{\mathbf{\Psi}}_{R}$ in (16) to write

$$
\begin{aligned}
\hat{\mathbf{\Psi}}_{R} & =\sum_{r=0}^{R} \frac{T^{r}}{r !} \mathbf{B}^{(r)} \hat{\mathbf{H}}_{R+1-r} \\
& =\sum_{r=0}^{R} \sum_{r^{\prime}=0}^{R+1-r} \frac{T^{r+r^{\prime}}}{r ! r^{\prime} !} \mathbf{B}^{(r)} \mathbf{H}^{\left(r^{\prime}\right)} .
\end{aligned}
$$

Let us pose $\tilde{r}=r+r^{\prime}$, we get

$$
\begin{aligned}
& \hat{\mathbf{\Psi}}_{R}=\sum_{r=0}^{R} \sum_{\tilde{r}=r}^{R+1} \frac{T^{\tilde{r}}}{r !(\tilde{r}-r) !} \mathbf{B}^{(r)} \mathbf{H}^{(\tilde{r}-r)} \\
& =\sum_{r=0}^{R+1} \sum_{\tilde{r}=r}^{R+1} \frac{T^{\tilde{r}}}{r !(\tilde{r}-r) !} \mathbf{B}^{(r)} \mathbf{H}^{(\tilde{r}-r)}-\frac{T^{R+1}}{(R+1) !} \mathbf{B}^{(R+1)} \mathbf{H}^{(0)} \\
& =\sum_{\tilde{r}=0}^{R+1} \frac{T^{\tilde{r}}}{\tilde{r} !} \sum_{r=0}^{\tilde{r}} \frac{\tilde{r} !}{r !(\tilde{r}-r) !} \mathbf{B}^{(r)} \mathbf{H}^{(\tilde{r}-r)}-\frac{T^{R+1}}{(R+1) !} \mathbf{B}^{(R+1)} \mathbf{\Lambda}^{(0)} .
\end{aligned}
$$

We can use (19) to simplify the last expression to

$$
\begin{aligned}
\hat{\mathbf{\Psi}}_{R} & =\sum_{\tilde{r}=0}^{R+1} \frac{T^{\tilde{r}}}{\tilde{r} !} \delta_{\tilde{r}} \mathbf{I}_{N_{T} M}-\frac{T^{R+1}}{(R+1) !} \mathbf{B}^{(R+1)} \boldsymbol{\Lambda}^{(0)} \\
& =\mathbf{I}_{N_{T} M}-\frac{T^{R+1}}{(R+1) !} \mathbf{B}^{(R+1)} \boldsymbol{\Lambda}^{(0)}
\end{aligned}
$$

If, again, we define the error matrix $\boldsymbol{\Theta}_{R}=\hat{\mathbf{B}}_{R} \mathbf{H}-\hat{\mathbf{\Psi}}_{R}$ and using the previous expression for $\hat{\mathbf{\Psi}}_{R}$, we find that

$$
\begin{aligned}
& \mathbf{E}_{R, d}=\left(\hat{\mathbf{\Psi}}_{R}+\boldsymbol{\Theta}_{R}-\mathbf{I}_{N_{T} M}\right)\left(\hat{\mathbf{\Psi}}_{R}+\boldsymbol{\Theta}_{R}-\mathbf{I}_{N_{T} M}\right)^{H} \\
& =\frac{T^{2 R+2}}{((R+1) !)^{2}} \mathbf{B}^{(R+1)} \boldsymbol{\Lambda}^{(0)}\left(\mathbf{B}^{(R+1)} \boldsymbol{\Lambda}^{(0)}\right)^{H}+\boldsymbol{\Theta}_{R} \boldsymbol{\Theta}_{R}^{H} \\
& -\frac{T^{R+1}}{(R+1) !} \mathbf{B}^{(R+1)} \boldsymbol{\Lambda}^{(0)} \boldsymbol{\Theta}_{R}^{H}-\frac{T^{R+1}}{(R+1) !} \boldsymbol{\Theta}\left(\mathbf{B}^{(R+1)} \boldsymbol{\Lambda}^{(0)}\right)^{H},
\end{aligned}
$$

where we can identify the expression of $\hat{\mathbf{E}}_{R, d}$ given in Corollary 1. Using Lemma 2 , we have that, as $T \rightarrow 0$, $\left\|\boldsymbol{\Theta}_{R}\right\|=O\left(T^{R+1}\right)$. Hence, we can conclude that

$$
\left\|\mathbf{E}_{R, d}-\hat{\mathbf{E}}_{R, d}\right\|=O\left(T^{2 R+2}\right) .
$$

\section{REFERENCES}

[1] W. G. Jeon, K. H. Chang, and Y. S. Cho, "An equalization technique for orthogonal frequency-division multiplexing systems in time-variant multipath channels," IEEE Transactions on Communications, vol. 47, no. 1, pp. 27-32, Jan 1999.

[2] L. Rugini, P. Banelli, and G. Leus, "Simple equalization of time-varying channels for OFDM," IEEE Communications Letters, vol. 9, no. 7, pp. 619-621, July 2005.

[3] T. Hrycak and G. Matz, "Low-complexity time-domain ICI equalization for OFDM communications over rapidly varying channels," in Fortieth Asilomar Conference on Signals, Systems and Computers, Oct 2006, pp. 1767-1771.

[4] M. Guillaud and D. T. M. Slock, "Channel modeling and associated inter-carrier interference equalization for OFDM systems with high doppler spread," in IEEE International Conference on Acoustics, Speech, and Signal Processing, 2003. Proceedings. (ICASSP '03)., vol. 4, Apr 2003, pp. IV-237.

[5] P. Schniter, "Low-complexity equalization of OFDM in doubly selective channels," IEEE Transactions on Signal Processing, vol. 52, no. 4, pp. 1002-1011, Apr 2004.

[6] K. Fang, L. Rugini, and G. Leus, "Low-complexity block turbo equalization for OFDM systems in time-varying channels," IEEE Transactions on Signal Processing, vol. 56, no. 11, pp. 5555-5566, Nov 2008.

[7] A. F. Molisch, M. Toeltsch, and S. Vermani, "Iterative methods for cancellation of intercarrier interference in OFDM systems," IEEE Transactions on Vehicular Technology, vol. 56, no. 4, pp. 2158-2167, Jul 2007.

[8] T. Zemen and C. F. Mecklenbrauker, "Time-variant channel equalization via discrete prolate spheroidal sequences," in The Thrity-Seventh Asilomar Conference on Signals, Systems Computers, 2003, vol. 2, Nov 2003, pp. 1288-1292.

[9] I. Barhumi and M. Moonen, "MLSE and MAP equalization for transmission over doubly selective channels," IEEE Transactions on Vehicular Technology, vol. 58, no. 8, pp. 4120-4128, Oct 2009.

[10] T. Hrycak, S. Das, G. Matz, and H. G. Feichtinger, "Low complexity equalization for doubly selective channels modeled by a basis expansion," IEEE Transactions on Signal Processing, vol. 58, no. 11, pp. 5706-5719, Nov 2010.

[11] E. Panayirci, H. Senol, and H. V. Poor, "Joint channel estimation, equalization, and data detection for OFDM systems in the presence of very high mobility," IEEE Transactions on Signal Processing, vol. 58, no. 8, pp. 4225-4238, Aug 2010.

[12] H. Senol, E. Panayirci, and H. V. Poor, "Nondata-aided joint channel estimation and equalization for OFDM systems in very rapidly varying mobile channels," IEEE Transactions on Signal Processing, vol. 60, no. 8, pp. 4236-4253, Aug 2012. 
[13] H. Hijazi and L. Ros, "Polynomial Estimation of Time-Varying Multipath Gains With Intercarrier Interference Mitigation in OFDM Systems," IEEE Transactions on Vehicular Technology, vol. 58, no. 1, pp. 140-151, Jan 2009.

[14] J.-P. M. G. Linnartz and A. Gorokhov, "New equalization approach for OFDM over dispersive and rapidly time varying channel," in 11th IEEE International Symposium on Personal Indoor and Mobile Radio Communications, PIMRC 2000, vol. 2, Sep 2000, pp. 1375-1379.

[15] A. Gorokhov and J.-P. M. G. Linnartz, "Robust OFDM receivers for dispersive time-varying channels: equalization and channel acquisition," IEEE Transactions on Communications, vol. 52, no. 4, pp. 572-583, Apr 2004.

[16] G. Li, H. Yang, L. Cai, and L. Gui, "A low-complexity equalization technique for OFDM system in time-variant multipath channels," in 2003 IEEE 58th Vehicular Technology Conference. VTC 2003-Fall, vol. 4, Oct 2003, pp. 2466-2470.

[17] A. Bourdoux, F. Horlin, E. Lopez-Estraviz, and L. V. D. Perre, "Practical channel estimation for OFDM in time-varying channels," in IEEE Globecom 2006, Nov 2006, pp. 1-5.

[18] S. Yerramalli, M. Stojanovic, and U. Mitra, "Partial FFT demodulation: A detection method for highly doppler distorted OFDM systems," IEEE Transactions on Signal Processing, vol. 60, no. 11, pp. 5906-5918, Nov 2012.

[19] J. Han, L. Zhang, and G. Leus, "Partial FFT demodulation for MIMOOFDM over time-varying underwater acoustic channels," IEEE Signal Processing Letters, vol. 23, no. 2, pp. 282-286, Feb 2016.

[20] A. F. Molisch, Wireless communications. John Wiley \& Sons, 2012, vol. 34 .

[21] Z. Tang, R. C. Cannizzaro, G. Leus, and P. Banelli, "Pilot-Assisted TimeVarying Channel Estimation for OFDM Systems," IEEE Transactions on Signal Processing, vol. 55, no. 5, pp. 2226-2238, May 2007.

[22] L. Rugini, P. Banelli, and G. Leus, "Chapter 7 - ofdm communications over time-varying channels," in Wireless Communications Over Rapidly Time-Varying Channels, F. Hlawatsch and G. Matz, Eds. Oxford: Academic Press, 2011, pp. 285 - 336. [Online]. Available: http://www.sciencedirect.com/science/article/pii/B9780123744838000078

[23] P. Duhamel and M. Vetterli, "Fast Fourier transforms: a tutorial review and a state of the art," Signal Proces., vol. 19, no. 4, pp. 259-299, 1990.

[24] R. A. Adams, A complete course calculus. Pearson Addison Wesley, 2006.

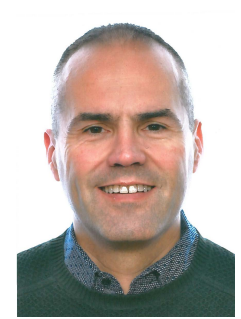

Xavier Mestre (S'96M'03SM'09) received the MS and $\mathrm{PhD}$ in Electrical Engineering from the Technical University of Catalonia (UPC) in 1997 and 2002 respectively and a Degree in Mathematics in 2011 by the same University. From January 1998 to December 2002, he was with UPC's Communications Signal Processing Group, where he worked as a Research Assistant. In January 2003 he joined the Telecommunications Technological Center of Catalonia (CTTC), where he currently holds a position as a Senior Research Associate and head of the Advanced Signal and Information Processing Department. He is author of 9 book chapters, 41 international journal papers and more than 100 articles in international conferences. He has been associate editor (2008-11, 2015-2019) and senior associate editor (2019-present) of the IEEE Transactions on Signal Processing. He is IEEE Senior member, elected member of the IEEE Sensor Array and Multi-channel Signal Processing technical committee (2013-2018), vice-chair of the EURASIP Special Area Team (SAT) on Theoretical and Methodological Trends in Signal Processing (2015-present) and member of the SAT on Signal Processing in Communications (2018-present). He has participated in the organization of multiple conferences and scientific events, such as the IEEE Wireless Communications and Networking Conference 2018 (general vice-chair), the IEEE International Symposium on Power Line Communications (technical chair), the European Wireless 2014 (general cochair), the European Signal Processing Conference 2011 (technical chair). He is general chair of the IEEE International Conference on Acoustics, Speech and Signal Processing 2020.

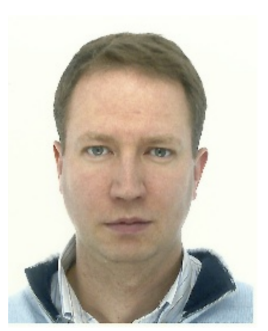

François Horlin (S'01M'02) received the electrical engineering degree and the Ph.D. degree from the Université catholique de Louvain (UCL), Louvainla-Neuve, Belgium, in 1998 and 2002 respectively. During his studies, he specialised in the field of digital signal processing for communications. In 2002, he joined the Inter-university Micro-Electronics Center (IMEC), Leuven, Belgium. He led the project aiming at developing a fourth-generation wireless communication system in collaboration with Samsung Korea. In 2007, Franois Horlin became associate professor at the Université libre de Bruxelles (ULB), Brussels, Belgium. Since 2014, he is full professor. He is currently giving three lectures in the field of digital telecommunications and is advisor of $6 \mathrm{Ph} . \mathrm{D}$. students (plus 13 already defended Ph.D. theses). He is author of a book, author of a book chapter, co-author of two patents, author or co-author of more than 180 publications in well-recognised journals and conferences. He currently chairs the IEEE signal processing chapter of the Benelux.

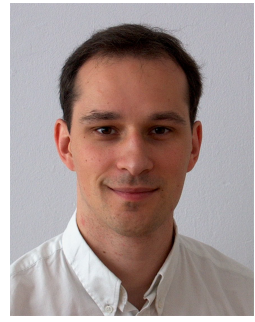

Jérôme Louveaux (S'98-M'02) received the electrical engineering degree and the $\mathrm{Ph}$. D. degree from the Universit catholique de Louvain (UCL), Louvain-la-Neuve, Belgium in 1996 and 2000 respectively. From 2000 to 2001, he was a visiting scholar in the Electrical Engineering department at Stanford University, CA. From 2004 to 2005, he was a postdoctoral researcher at the Delft University of technology, Netherlands. Since 2006, he has been a Professor in the ICTEAM institute at UCL. His research interests are in signal processing for digital communications, and in particular: multicarrier modulations, xDSL systems, resource allocation, synchronization and estimation. Prof. Louveaux was a corecipient of the "Prix biennal Siemens 2000" for a contribution on filter-bank based multi-carrier transmission and co-recipient of the the "Prix Scientifique Alcatel 2005" for a contribution in the field of powerline communications.$$
\text { A }
$$

\footnotetext{
participated to various national, European and international projects. From 2015 to 2018 , he has been a regular visitor and actively collaborates with the Centre Tecnolgic Telecomunicacions Catalunya (CTTC), Castelldefels, Spain and National Institute of Information and Communications Technology

(NICT), Tokyo, Japan.

François Rottenberg (S'15M'18) received the M.Sc. in electrical engineering from the Université 2014, and the Ph.D. degree from UCL and Universite doctoral researcher with the University of Southe California (USC), Los Angeles, U.S.A, leading the massive MIMO project aiming at demonstrat
} 